\title{
The Diagnostic Value of Color Doppler Ultrasound in Ureteral Calculi
}

\author{
Fan Weibo \\ Department of Ultrasound, Jingzhou Central Hospital, The Second Clinical Medical College, Yangtze University, Jingzhou, China

\section{Email address:} \\ 176497992@qq.com

\section{To cite this article:} \\ Fan Weibo. The Diagnostic Value of Color Doppler Ultrasound in Ureteral Calculi. International Journal of Medical Imaging. \\ Vol. 6, No. 2, 2018, pp. 12-17. doi: 10.11648/j.jimi.20180602.11
}

Received: July 21, 2018; Accepted: August 1, 2018; Published: August 30, 2018

\begin{abstract}
Ureteral calculi are one of the most common acute abdomen, most of which are from renal calculi. Color Doppler ultrasound plays a very important role in the diagnosis of ureteral calculi, and has the advantages of simplicity, economy, non-invasive, no radiation, and repeatable examination. The purpose of this study is to investigate the clinical value of color Doppler ultrasound in the diagnosis of ureteral calculi. To achieve this goal, a retrospective analysis was performed to analyze the clinical symptoms, ultrasonographic appearances and results of 112 patients with ureteral calculi who were treated in author's hospital from March 2016 to March 2018. It showed that there were 5 cases of bilateral ureteral calculi, 59 cases of left ureteral calculi, 48 cases of right ureteral calculi, 42 cases of upper ureteral calculi, 11 cases of middle ureteral calculi and 59 cases of lower ureteral calculi in the results of the 112 patients with ureteral calculi. As ultrasound examination can show the location, number and size of the calculi, which can help clinical treatment and can be used as the first choice for ureteral calculi. Therefore, it is concluded that ultrasound diagnosis of ureteral calculi has the advantages of painless, non-invasive, convenient and easy to operate repeatedly, and can be used as the first choice for checking ureteral calculi.
\end{abstract}

Keywords: Color Doppler Ultrasound, Ureteral Calculi, Diagnostic Value

\section{Introduction}

In recent years, with the continuous development of ultrasound equipment and the improvement of the diagnostic level of technicians, the detection rate of ureteral calculi by ultrasound has been relatively improved. At the same time, CT, KUB and IVU are not suitable for repeated inspections because of the high price and radiation. After all, ultrasound is non-invasive, non-radiative, and can be repeatedly tested. The advantage of ultrasonography is that it can clearly show the $\mathrm{X}$-ray negative calculi in the ureter, which compensates for the deficiency of X-ray examination to varying degrees. In the course of ultrasound imaging, the degree of hydro-nephrosis due to ureteral obstruction can be known, so that renal function can be estimated generally, and other diseases of the urinary system coexisting with ureteral calculi can also be found at the same time.

Despite this, during the ultrasound imaging process, it is easy to cause missed diagnosis of ureteral calculi due to the physical characteristics of ultrasound and other reasons. The reasons are as follows: 1 . The patient's obesity and the interference of intestinal contents can make the sonogram blurred, leading to misdiagnosis. The patients' huge body and old age may result in poorer penetrating ultrasound and an unclear strong echo structure of the calculi. The patients' poor intestinal condition may influence the penetration of the ultrasound in the ureteral area, which can be solved by allowing the patient to be re-examined after bowel preparation. 2. Due to the small ureteral calculi or short-term obstruction, there is no obvious accumulation of water in the renal pelvis and ureter, ultrasound is not found and easily leads to misdiagnosis. 3. The patients often have an acute onset, poor bladder-filling, and difficulty in cooperating with pain, which is also one of the reasons for misdiagnosis. Therefore, for patients with high suspicion of ureteral calculi and undetectable ultrasound examinations, abdominal plain films, CT or retrograde pyelography can be performed to avoid misdiagnosis of ureteral calculi [1].

Ureteral calculi are one of the most common acute abdomen, most of which are from renal calculi. The stimulation to the ureteral wall from calculi can cause local visceral mucosal 
damage, edema and concurrent inflammation, causing ureteral obstruction and spasmodic contraction, leading to dull or paroxysmal abdominal pain, accompanied with gross or microscopic hematuria. Ureteral calculi can cause urinary tract obstruction and different degrees of hydrocele in the affected kidney. In severe cases, the renal cortex becomes thinner, its function is lost or even get uremia. The clinical data of 112 patients who were treated in author's hospital from March 2016 to March 2018 were analyzed and reported as follows.

\section{Materials and Methods}

\subsection{Clinical Data}

This clinical study selected 112 patients from March 2016 to March 2018 in author's hospital, which include 70 males and 42 females aged 16-72 years, mean age (38.2). \pm 11.3 ) years old. Those patients are accompanied by sudden, intermittent lumbar pain, radiating along the ipsilateral ureter to the lower abdomen, perineum, external genitalia and inner thighs, and often accompanied by nausea, vomiting, microscopic hematuria or even gross hematuria. They were confirmed by surgery, extracorporeal shock wave lithotripsy and self-extracting calculi.

\subsection{Instruments and Methods}

The PHILIPS IU22 ultrasound diagnostic instrument is used, and the $3.5-5 \mathrm{MHz}$ convex array probe is routinely used. Patients with upper ureteral calculi were placed in the lateral or prone position, and the probe was placed in the lateral lumbar region with the renal hilum or renal pelvis as the sign showing the ureteropelvic junction. Patients with the second stenosis calculi were placed in the supine position. The probe was obliquely cut in the lower abdomen and the iliac artery was found by compression. The dilated ureter was found in front of the iliac artery, and the probe was used to display the long axis of the ureter. Patients with lower ureteral calculi were placed in supine position, whose bladder was moderately filled and the probe was pressurized. Then the lower ureteral opening was scanned in the trigone of the bladder, and the scanning was performed upward. By following a certain examination sequence, combined with a variety of examination methods, multi-position and multi-angle scan, and abdominal ultrasound can be done to patients with ureteral calculi. It can also improve the detection rate of ureteral urinary calculi by some scanning methods like moderate bladder-filling, diuretic pressurization and adding high-frequency probes [2].

\section{Results}

In this group of 112 patients, 36 cases of upper ureteral calculi, 5 cases of middle calculi, and 45 cases of lower ureteral calculi were detected by multi-position and multi-angle scanning of abdominal ultrasound. A total of 86 cases were detected, and the detection rate was $76.8 \%$. Seven cases of ureteral calculi were detected by diuretic pressurization method, and 15 cases of pelvic and intra-ureteral calculi were detected by moderate bladder-filling method. There were 108 cases of ureteral calculi were detected by multiple checking methods in this group, and the detection rate was $96.4 \%$. There were 4 cases of misdiagnosis couldn't be detected because of the patient's obesity and flatulence interference. The diagnosis only indicated the separation of renal sinus echo, but the upper limit of upper ureteral diameter was normal. There were 101 patients $(90.2 \%)$ who had different degrees of hydronephrosis in this group of patients with ureteral calculi. Eleven patients had normal renal function and had no obvious separation in the collecting system. The diameter of the calculi is $4-14 \mathrm{~mm}$, which is more commonly seen in $5-8 \mathrm{~mm}$.

\section{Discussion}

Starting from the renal pelvis to the bladder triangle, the ureter is about $20-34 \mathrm{~cm}$ long that located in the posterior peritoneum. There are three physiological stenosis parts in the ureter, which are separately located at the junction of the renal pelvis and ureter, the site that ureter spans the iliac vessels and the ureteral bladder wall. Calculi can often stay in these stenotic sites. Most ureteral calculi are derived from renal calculi, and primary calculi are rare. The ureteral calculi can cause urinary tract obstruction, and lead to different degrees of hydrocele in the affected renal. The higher the position of ureteral calculi, the more severe the degree of obstruction and hydro-nephrosis. When the sonogram shows the hydro-nephrosis, checking along with the dilated ureter, it can be seen that the dilated ureter is suddenly interrupted, and the strong echo and the wall boundary is clearly defined, followed by an acoustic shadow. Because of the different size, shape, location and composition of ureteral calculi, their sonograms are also displaying differently. For example, the uric acid calculi has a loose texture and rough surface, which presents a round or elliptical high echogenic area and the acoustic shadow behind it is weak or not obvious. The calcium oxalate calculi, possessing hard texture and smooth surface, the sonogram of which can only show that its surface profile presents like an arc-shaped high echogenic area and there is an acoustic shadow at the rear. The small stone with a rough surface has a strong echo of point, and there is no obvious acoustic shadow.

The typical sonogram of ureteral calculi appears as a calculus echo at the distal end of the dilated ureter, which is characterized by a curved strong light band, with a acoustic shadow behind the ureter, and a ureteral dilatation above the calculi obstruction (see Figure 1), hydronephrosis, and the inner diameter of the ureter of far end is significantly smaller than the proximal end [3]. 


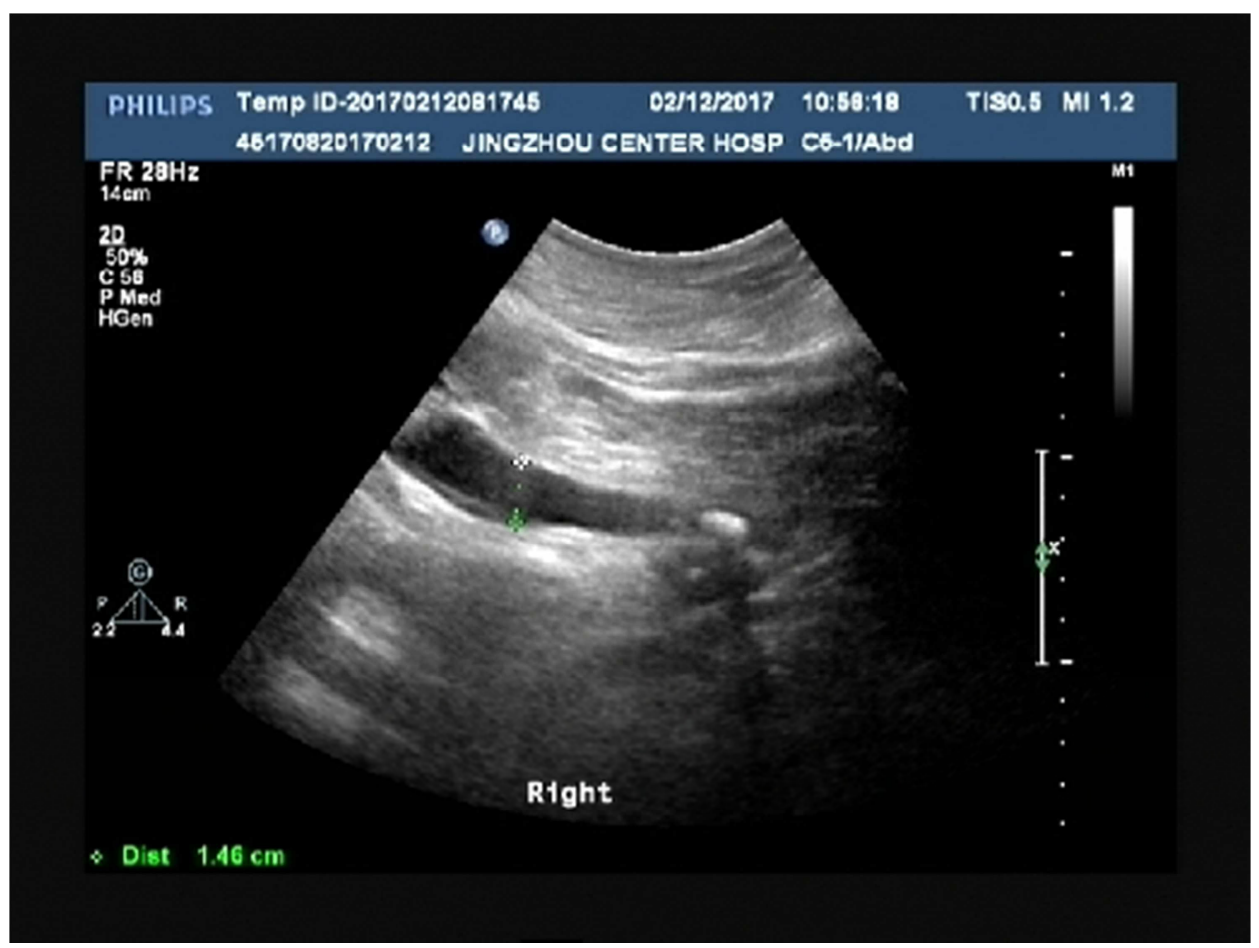

Figure 1. Upper ureteral calculi on the right side.

To detect ureteral calculi quickly and accurately, you must be familiar with the anatomy and characteristics of the ureter, and use reasonable timing and examination sequence to save time and diagnose quickly. The ureteral calculi are best examined during an acute attack of renal colic, because at this time the ureter is mostly accompanied by dilatation, and it is easier to find the obstruction site when the bladder is not full.

According to the literature cited [4]: the inner segment of the bladder wall is the narrowest part of the ureter. With its unique dynamic characteristics, $70 \%$ of the ureteral stones are located at this site [5]. During the examination, the inner segment of the bladder wall is first found to achieve a rapid diagnosis. If no stone echoes is found in the inner segment of the bladder wall, then the hydronephrosis should be checked. If there is, a hydroureterosis should be confirmed from the hydronephrosis to the pelvic ureter junction, then the multi-faceted scan of the abdomen, the back and the lateral waist will allow for the examination of most of the calculi. If no stones are found in the first and the third stenosis, then the second stenosis should be scanned, during which the supine position is usually taken. The common iliac artery is first found, and the long axis of the ureter is found in front of the common iliac artery. Scanning below and following the hydroureterosis can assure the detection of calculi. The calculi can also be found by firstly being examined by the bladder, which shows the opening of bilateral ureters in the bladder wall, and then scan up.

During the examination of ureteral calculi, if the sonographer can master a variety of ultrasound diagnostic techniques for ureteral calculi, the display rate of ureteral calculi can be more effectively improved: 1 . The locating scan can be done based on the most painful location of the patient, which can reduce the blind scanning time, especially for patients with no hydronephrosis and no obvious ureteral dilatation, and it is not easy to track each segment of the ureter. 2. For some patients who has no obvious pain symptoms, let them to fill the bladder moderately and increase the degree of dilatation of the ureter as well as appropriately reduce the gain, pressurization, multi-position and multi-section, trying to make the sound beam perpendicular to the ureter that makes it easy to find smaller stones. 3. It has been reported that intracavity ultrasound has obvious advantages in the examination of lower ureteral calculi, especially for patients with acute abdomen and less bladder filling [6].

Because of the obvious bladder irritation, not easy to urinate, small calculi and ureter without dilatation, obesity, intestinal contents and gas interference, etc., patients who have lower ureteral calculi can affect the diagnosis of lower ureteral calculi by abdominal ultrasound, while intracavity ultrasound requires less filling of the bladder, and the diagnosis of calculi is more sensitive than the abdomen. However, patients with renal calculi often appear in the emergency department at night, so it is not convenient to carry out intracavity ultrasound. Therefore, intracavity ultrasound has not been used in author's hospital to diagnose ureteral calculi. 4. Ultrasonic diagnosis of ureteral calculi with diuretic compression method is convenient and quick to check. The patient has no noticable discomfort and adverse reactions and is easy to accept. Check again by using the diuretic abdominal compression method (use the sandbag to compress the second stenosis of the ureter closest to the abdominal wall), cleaning the enema, using the diuretic and filling the bladder, forming a good urine-stone acoustic interface, which helps to improve the calculi detection rate. 5. On the basis of two-dimensional ultrasound examination, color Doppler imaging can be used to identify 
the dilated ureter and surrounding blood vessels, and the "twinkling artifact" of color Doppler ultrasound can be used to diagnose ureteral calculi [7-8]. "Twinkling artifact" refers to the appearance of a flashing blood flow signal (similar to the "tail of a comet" sign) behind the ureteral calculi (see Figures 2 and 3$)$.

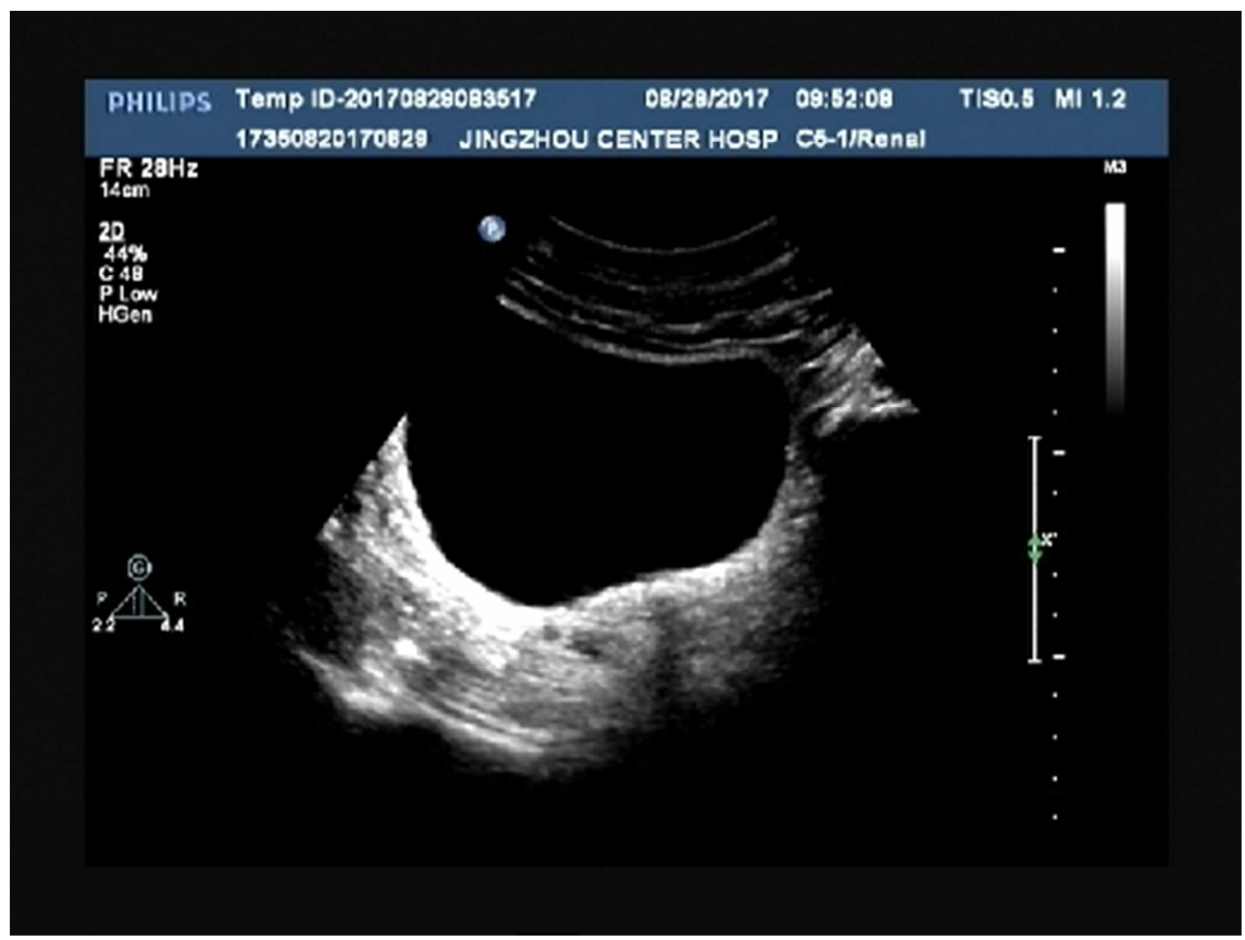

Figure 2. Inner bladder wall ureteral calculi on the left side.

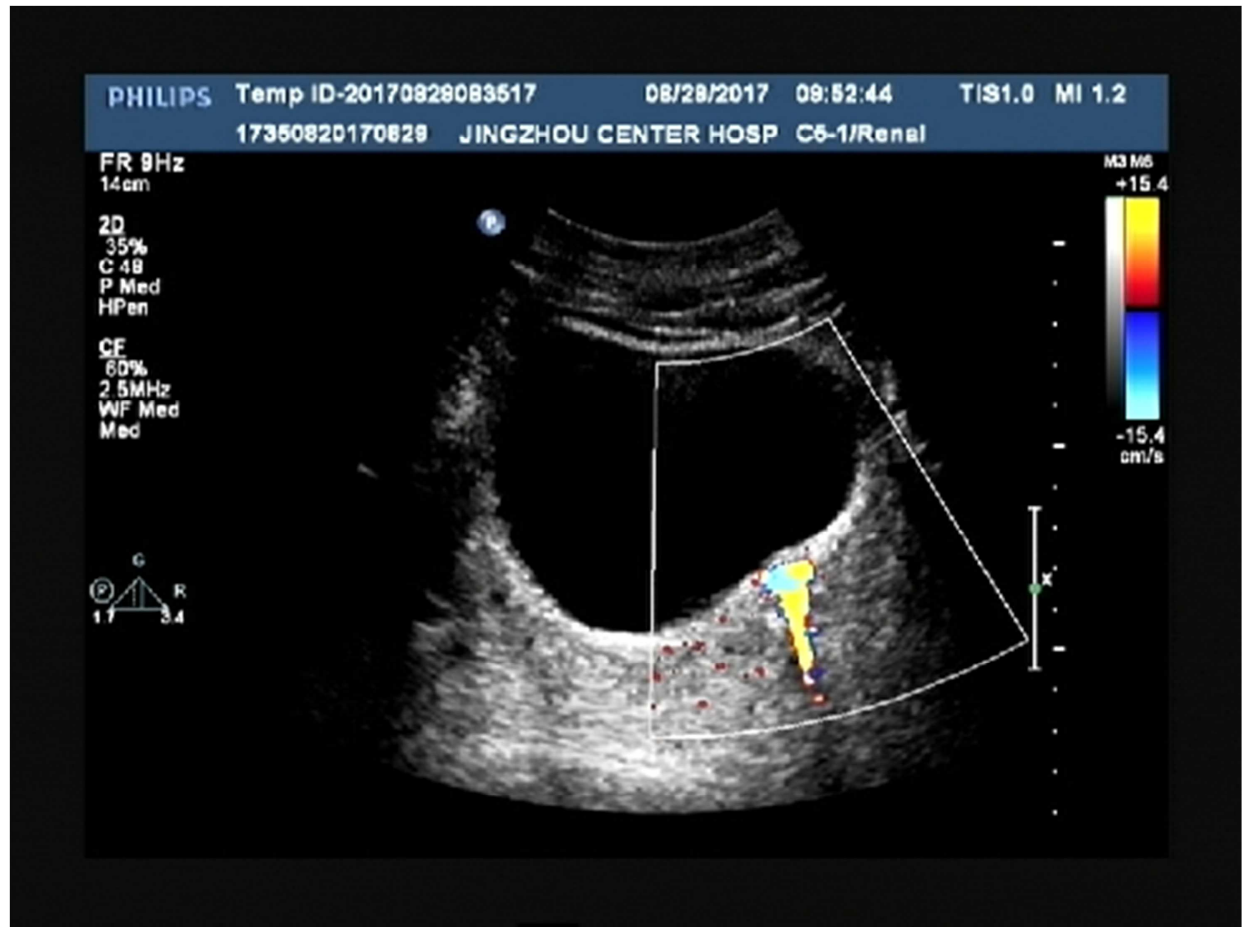

Figure 3. The "twinkling artifact" of inner bladder wall ureteral calculi on the left side.

This feature can be used as a basis for the presence of ureteral calculi. Based on the mechanism of twinkling artifact, a variety of hyperechoic structures can show twinkling artifact, such as ureteral calcification, foreign body, ureteral stent, double "J" tube and intestinal gas, so doctors should also pay attention to identify these flashes during the examination. The interference of artifacts on the diagnosis of calculi. 6. Try to use a higher frequency probe scanning (except for obesity). The local enlargement can clearly show the ureteral lumen and display $2 \mathrm{~mm}$ tiny calculi in the ureter to improve the display 
rate of ureteral calculi. 7. Perirenal fluid collection (see Figure 4 ) is an indirect sign of acute urinary tract obstruction caused by partial ureteral calculi [9-10], the side of the perirenal fluid collection that has water accumulation or renal sinus echo separation is more obvious than the contralateral side.

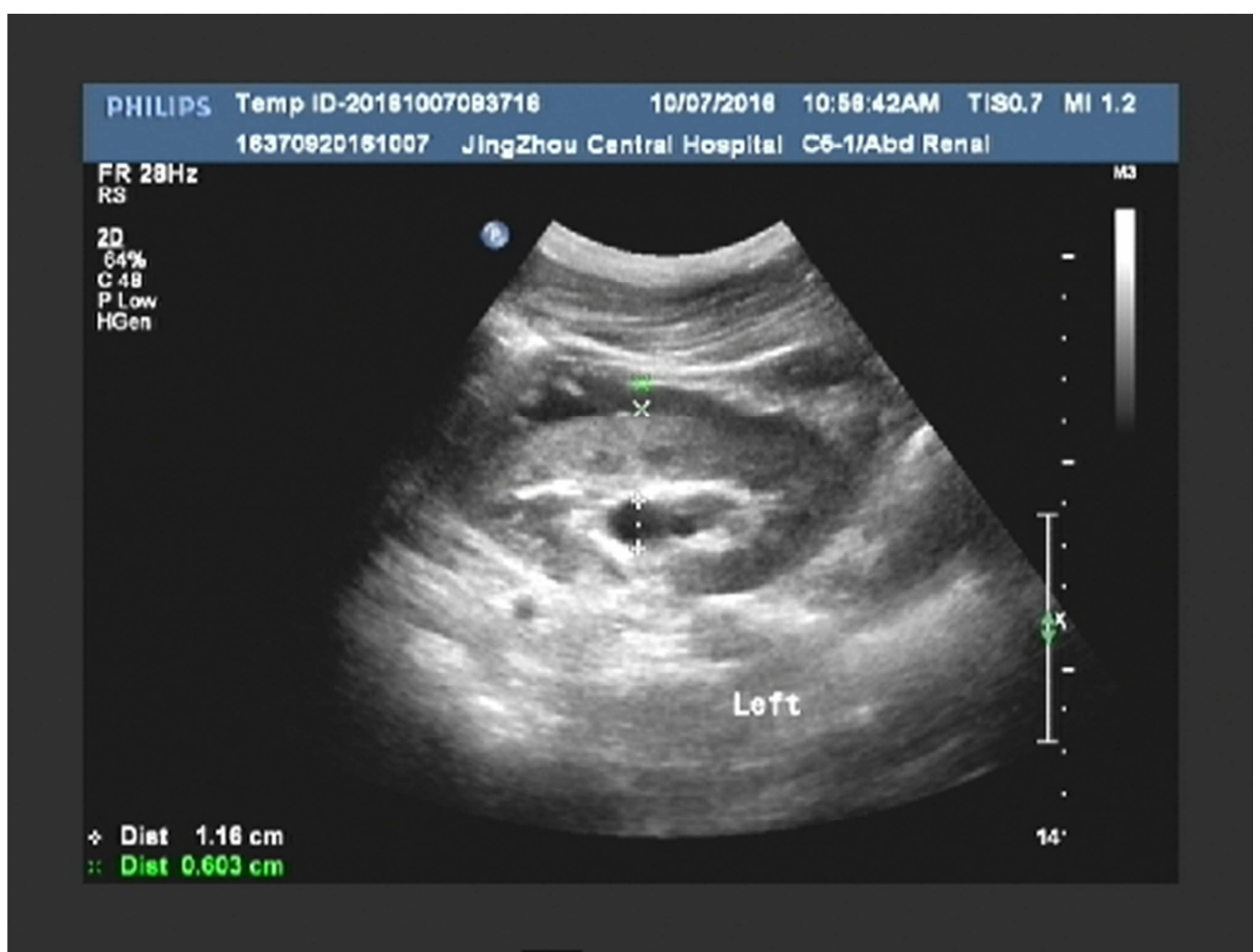

Figure 4. Mild hydronephrosis of the left renal with perirenal fluid collection.

The clinical symptoms are typical and there are red blood cells in the urine test. The urinary calculi should be highly suspected and the bladder should be properly filled if necessary. For those who don't have hydronephrosis, ureteral calculi are easily overlooked and further missed. 8. Add tissue harmonic, which can darken the ureteral lumen and enhance the echo of the wall, and not only can it clearly show the ureteral calculi echo, boundary and posterior acoustic shadow, but also clearly show the ureteral lumen with different degrees of expansion.

If the ultrasound doctors have mastered the examination sequence of ureteral calculi, combined with a variety of examination methods, the location, size, shape and number of ureteral calculi can quickly and accurately found, providing a strong basis for clinical treatment, which is a safe, fast, effective, high-rate diagnostic inspection.

\section{Conclusion}

The Previous literature reported that multi-slice spiral CT diagnosis of calculus sensitivity and specificity was close to $100 \%$ [11], and the sensitivity of ultrasound diagnosis of ureteral calculi was $19 \%$ to $93 \%$ [12], higher than KUB. The sensitivity of B-ultrasound diagnosis of hydro-nephrosis was $72.6 \%$ and the specificity was $73.3 \%$ [13]. The study concluded that the sensitivity of ultrasound diagnosis of hydro-nephrosis was $93.5 \%$, which was higher than the sensitivity of detected calculi. In the presence of hydrocele, the sensitivity of B-ultrasound was increased from $72.0 \%$ to
91.2\%. As an important indicator of post-renal obstruction, hydro-nephrosis helps emergency doctors to find ureteral calculi [14]. It is clear that the risk factors associated with the degree of hydrocele can help to predict calculi information and guide the next treatment decision.

In summary, color Doppler ultrasound plays a very important role in the diagnosis of ureteral calculi, and has the advantages of simplicity, economy, non-invasive, no radiation, and repeatable examination. Ultrasound examination can show the location, number and size of the calculi, which can help clinical treatment and can be used as the first choice for ureteral calculi.

\section{References}

[1] Shen Long. Comparison of the Value of Ultrasound and CT in the Diagnosis of Ureteral Calculi $[\mathrm{J}]$. Contemporary Medicine Forum, 2017, 15 (8):54-55.

[2] Yang Xiaoxia, Zhou Xiaoying. Value Analysis of Color Doppler Ultrasound in Diagnosis of Ureteral Calculi [J]. Clinical Medicine \& Engineering, 2015, 22 (11):1414-1415.

[3] Lu Ming, Luo Mei, Sun Yu. Diagnostic Value of Ultrasound for Ureteral Calculi [J]. Xinjiang Medical Journal, 2015, 45 (10):1516-1517.

[4] Jing Fengjie, Li Jinyan. Clinical Value of Ultrasound in Diagnosis of Ureteral Calculi [J]. Chinese Medical Journal of Metallurgical Industry, 2016, 33 (2):200-201. 
[5] WOLF J S Jr. Treatment selection and outcomes:ureteral calculi [J]. Urol Clin North Am, 2007, 34:421-430.

[6] Jiang Ju, Gao Jicheng, Zhou Chunyan, et. al. Comparative Analysis of Intracavitary Ultrasound and Transabdominal Ultrasound in the diagnosis of Ureteral Calculi [J]. Journal of Hebei Medical University, 2014, 35 (3):294-296.

[7] Cui Chuanxiang. The Application Value of Color Doppler Flash Artifact in the Diagnosis of Ureteral Calculi [J]. Huaihai Medicine, 2015, 33 (6):554-555.

[8] Wu Jianhui, Meng Hongzhou, He Yi. The Value of Color Doppler Ultrasound Scintillation Artifacts in Ultrasonic Localization of Extracorporeal Shock Wave Lithotripsy [J]. China Modern Doctro, 2016, 54 (25):15-18.

[9] Wen Xingyue. Ultrasound Observation and Analysis of Ureteral Calculi with Acute Perirenal Effusion. Journal of Clinical Medical Literature, 2014, 1 (7):1197-1201.

[10] Yang Yanzhen. Value of Ultrasound in the Diagnosis of Acute Ureteral Calculi Associated with Perirenal Effusion [J]. Clinical Journal of Chinese Medicine, 2017, 9 (8):69-72.
[11] Na Yanqun, Ye Zhangqun, Sun Yinghao, Sun Guang. Guidelines for the Diagnosis and Treatment of Urological Diseases in China. People's Medical Publishing House (PMPH), 2014.

[12] D P Viprakasit, M D Sawyer, S D Herrell, et a1. Limitations of ultrasonography in the evaluation of urolithiasis:a correlation with computed tomography. JEndourol, 2012, 26 (3):209 213.

[13] M K Herbst, G Rosenberg, B Daniels, et a1. Effect of provider experience on clinician-performed ultrasonography for hydronephrosis in patients with suspected renal colic. Ann Emerg Med, 2014, 64 (3):269-276.

[14] B Daniels, CP Gross, A Molinaro, et a1. STONE PLUS:Evaluation of Emergency Department Patients With Suspected Renal Colic, Using a Clinical Prediction Tool Combined With Point-of- Care Limited Ultrasonography. Ann Emerg Med, 2015. 